\title{
The first "Best Paper of Nuclear Medicine Review" session at the XIV International Congress of the Polish Society of Nuclear Medicine 28-30th of May 2014
}

This year's International Congress of the Polish Society of Nuclear Medicine (PSNM) greatest novelty was the Best Paper contest held under the patronage of PSNM President and Nuclear Medicine Editors. The winning authors were chosen among those who had received highest scores during abstract review. First prize was given to Beata Chrapko, Marcin Pachowicz, Anna Nocuń, Andrzej Głowniak and Maciej Wójcik for their study on the heart adrenergic system in patients with cardiac arrhythmias and end-stage renal disease. Second prize was awarded to Jolanta Kunikowska, Renata Matyskiel, Sadeh Totounchi, Laretta Grabowska, Łukasz Koperski, Maria Teresa Płazińska and Leszek Królicki, the authors of "18FDG PET/CT utility in the evaluation of adrenal tumors" manuscript. Andrzej Mazurek, Eugeniusz Dziuk, Zofia Stembrowicz-Nowakowska, Stanisław Piszczek, Agnieszka Giżewska and Mirosław Dziuk, the authors of
"SPECT/CT diagnostic algorithms in pulmonary embolism diagnosis", won third prize. The other finalists were Michał Maurin, Dariusz Pawlak, Piotr Garnuszek, Artur Jaroń, Nils Matzler-Nolte, Raphael Stoll and Renata Mikołajczak presenting the results of "Physicochemical and radiochemical characteristics of DOTA-gastrin (CP04) analogues complexes with radiometals" study and A. Syguła, Daria Handkiewicz-Junak, Kornelia Hasse-Lazar, Beata Jurecka-Lubieniecka, Aleksandra Ledwon, Agnieszka Kotecka and Barbara Jarząb, the authors of "Consolidation therapy with long-acting somatostatin analogues after radioisotope therapy in neuroendocrine tumors". The Special Prize for the congress best paper and the implementation of a new diagnostic technique was awarded to Alicja Hubalewska-Dydejczyk, Anna Sowa-Staszczak, Dorota Pach, Monika Tomaszuk, Agnieszka Stefańska, Monika Buziak-Bereza,

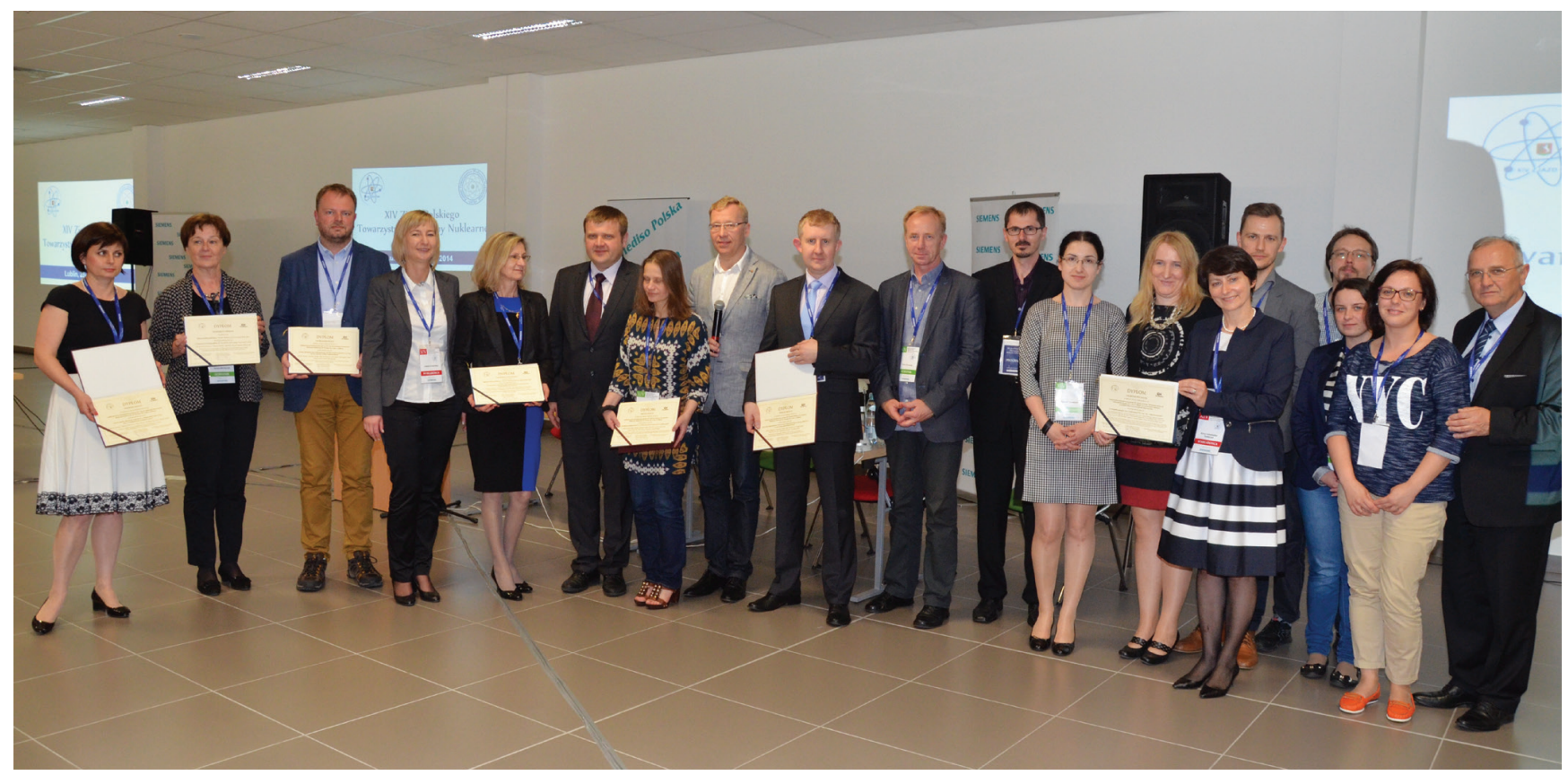

Figure 1. The Best Paper of Nuclear Medicine Review Award Winners together with the President of the Polish Society of Nuclear Medicine and the Editors of the journal. Lublin 2014 
Agata Jabrocka-Hybel, Aleksandra Gilis-Januszewska, Maciej Małecki, Tomasz Bednarczuk, Grzegorz Kamiński, Aldona Kowalska, Malgorzata Trofimiuk-Muldner, Renata Mikołajczak and Barbara Janota presenting 3-year results of ${ }^{99 \mathrm{~m}} \mathrm{Tc} /{ }^{111} \mathrm{In}$ GLP-1 analogue scintigraphy. All winning manuscripts will be published in Nuclear Medicine Review at the earliest.

This session also held the Best Polish NMR Publication 2011-2013 contest. The winning papers had the highest quotation number. First prize was awarded to Eftychia Koumarianou, Dariusz Pawlak, Agnieszka Korsak and Renata Mikołajczak for "Comparison of receptor affinity of natSc-DOTA-TATE versus nat Ga-DOTA-TATE". Second prize went to Marek Ruchała, Ewelina Szczepanek and Jerzy Sowiński for "Diagnostic value of radionuclide scanning and ultrasonography in thyroid developmental anomaly imaging", and third prize to Grażyna Łapińska, Małgorzata Bryszewska, Agnieszka Fijołek-Warszewska, Izabella Kozłowicz-Gudzińska, Paweł Ochman and Agata Sackiewicz-Słaby for "The diagnostic role of ${ }^{68} \mathrm{Ga}-\mathrm{DOTA}$-TATE PET/CT in the detection of neuroendocrine tumours".

We would like to congratulate the winners on their achievement. We also invite other authors to submit their research for publishing in Nuclear Medicine Review.

Professor Grzegorz Kamiński, Editor-in-Chief Jolanta Kunikowska, PhD, and Mirosław Dziuk, PhD, Deputy Editors 